\title{
An action sequence held in memory can interfere with response selection of a target stimulus, but does not interfere with response activation of noise stimuli
}

\author{
Paul S. Mattson and Lisa R. Fournier \\ Washington State University, Pullman, Washington
}

\begin{abstract}
Withholding an action plan in memory for later execution can delay execution of another action if the actions share a similar (compatible) action feature (e.g., response hand). We investigated whether this phenomenon, termed compatibility interference (CI), occurs for responses associated with a target as well as responses associated with distractors in a visual selection task. Participants planned and withheld a sequence of keypress responses (with their right or left hand), according to the identity of a stimulus (A), and then immediately executed a keypress response (with their right or left hand) to a second stimulus (B), according to the identity of a target letter appearing alone or among distractor letters. Distractor letters were either response compatible or incompatible with the target and appeared either simultaneously with the target (Experiments 1A and 2) or $100 \mathrm{msec}$ before the target (Experiment 1B). Also, stimulus-response mapping was either 1:1 (Experiment 1) or 2:1 (Experiment 2). Results showed that the response to the Stimulus B target was delayed when it required the same response hand as Stimulus A, as opposed to a different hand. Also, the target reaction time for Stimulus B was greater when the target was flanked by incompatible distractors than when it was flanked by compatible distractors. Moreover, the degree of CI was consistent across the compatible-, incompatible-, and no-distractor conditions, indicating that CI generalizes to responses associated with a target, but not to those associated with distractors. Thus, $\mathrm{CI}$ occurs at a response selection, not at a response activation stage. Implications for the code occupation account for CI (e.g., Stoet \& Hommel, 1999, 2002) and an alternative account for $\mathrm{CI}$ are discussed.
\end{abstract}

Everyday actions, such as turning on an appliance, starting your car, and programming your television remote, require the production of action plans. An action plan is a set of muscle commands that are structured before a movement sequence begins, allowing the entire sequence to be carried out (Keele, Cohen, \& Ivry, 1990). According to Jeannerod (1997), action planning requires that the appropriate motor schemas be selected, related to the proper internal and external cues, and organized into the appropriate sequence. Sometimes we have to momentarily suspend the execution of one action plan in order to execute another action that takes precedence. For example, we may plan a sequence of responses required to turn on a car air conditioner, but before we execute this action, we may execute another action to retrieve our sunglasses, which are sliding, along with other items, across the dashboard. An interesting question to ask is whether our ability to carry out an immediate action (such as retrieving our sunglasses) is affected by the action plan (to turn on the air conditioner) currently being held in memory. Recent research suggests that execution of an action, at least in some cases, can be adversely affected by an action plan currently being held in memory.

Stoet and Hommel (1999) showed that withholding an action plan in memory for later execution can sometimes delay the execution of another action. In their study, two different visual stimuli were presented sequentially. Participants were instructed to plan and withhold a sequence of keypresses with either their right or left hand with regard to the identity of the first stimulus (Stimulus A). While participants were withholding the action plan to Stimulus A, a second visual stimulus appeared (Stimulus B). An immediate keypress response with either the right or left hand, with regard to the identity of Stimulus B, was required. After executing a speeded response to Stimulus B, participants executed the planned action to Stimulus A. Results showed that the response to Stimulus B was longer when it required the same (compatible) hand than when it required a different (not compatible) hand from that required by Stimulus A. This delay in executing an action to the intervening stimulus (Stimulus B) when it shares a compatible feature with the action currently held in memory is referred to as compatibility interference (CI). 
To explain CI, Stoet and Hommel $(1999,2002)$ proposed the code occupation hypothesis $(\mathrm{COH})$, which was built on the foundations of the common coding hypothesis (CCH) developed by Prinz (1990, 1997; for an overview, see Hommel, Müsseler, Aschersleben, \& Prinz, 2001). The $\mathrm{COH}$ assumes that intentional actions are cognitively represented in terms of the proximal and distal effects that such actions produce; hence, these action-effect codes contain both our internal actions and the anticipated or perceived effects of the action in the environment (Hommel et al., 2001; Müsseler \& Hommel, 1997a). Action and perceptual features share a common representational domain that is consistent with the existence of mirror neurons (e.g., Gallese, Fadiga, Fogassi, \& Rizzolatti, 1996), as well as that of visuomotor neurons (e.g., Sakata, Taira, Murata, \& Mine, 1995; Taira, Mine, Georgopoulos, Murata, $\&$ Sakata, 1990). An action feature can be a movement direction or the side of the body in which the effectors used to carry out an action are located (e.g., a movement to the right, a movement with the right hand), and a perceptual feature can be the orientation or movement direction of a stimulus (e.g., a right-pointing arrow, a stimulus moving to the right). To specifically account for $\mathrm{CI}$, the $\mathrm{COH}$ assumes that all features (perceptual and action features) controlling the action plan are bound together into a common representation (action plan) that occupies the codes that represent it. This occupation of codes causes these codes to become temporarily less available for other actions or perceptions until the action plan is executed or abandoned (see also Prinz, 1997).

Thus, the $\mathrm{COH}$ predicts that a response will be delayed if there is partial feature-code overlap with the action plan held in memory, due to code occupation. For example, if an action sequence that requires the right hand is planned, a right code will be occupied, delaying any additional actions that require the right code (e.g., the right hand) until the planned action is performed or released; this was the result obtained by Stoet and Hommel (1999). In accordance with the COH, Stoet and Hommel (1999) found that CI occurred only when a sequence of actions had been planned in advance of the stimulus that required an immediate action. Furthermore, they found CI when effectors shared a side of the body (e.g., left hand and left foot, which share the left code), although the effect was not as pronounced as when only one effector (e.g., left hand) was used for both responses. Recent evidence by Wiediger and Fournier (in press) also shows that motor overlap is not sufficient for CI to occur, particularly when responses are based on stimulus detection, where cognitive demands required for the action event are relatively low and likely do not require the generation of feature codes. These findings suggest that it is not effector or motor overlap, but an overlap in the feature codes (e.g., right or left) that makes up the action plan that causes CI (see also Müsseler \& Hommel, 1997a, 1997b; Müsseler \& Wühr, 2002; Wühr \& Müsseler, 2001).

According to the $\mathrm{COH}$, CI should occur for an intervening speeded response whenever there is partial feature overlap between the intervening action and an action plan currently held in memory. However, because CI has only been investigated in visual tasks in which the intervening event consists of a single stimulus that is presented alone, it is not known whether CI will generalize to a selection task in which a task-relevant stimulus must be selected among other stimuli. Determining whether CI can generalize to a visual selection task is important, because most of our actions require that we select and respond to one stimulus that is embedded among other stimuli (e.g., as in our sunglasses example).

Also, because many stimuli we wish to ignore (distractors) are associated with actions that can compete with the action associated with the task-relevant stimulus (target), it is of great interest to us to determine whether CI generalizes to these competing actions as well. C. W. Eriksen and colleagues (Coles, Gratton, Bashore, Eriksen, \& Donchin, 1985; C. W. Eriksen \& Schultz, 1979) showed that when a target is closely surrounded by distractors, the response associated with the target and the response associated with the distractors are activated. If the response associated with the distractors is different (incompatible) than that associated with the target, the distractor response (which accumulates over time) will compete with the target response. As a result, the distractor response will have to be overridden (or inhibited) in order for one to respond correctly to the target. Overriding the competing, incorrect activity associated with the distractors takes time and, in turn, delays correct response execution to the target relative to when the target occurs alone or is accompanied by distractors that activate the same (compatible) response as the target (e.g., Coles et al., 1985; B. A. Eriksen \& C. W. Eriksen, 1974; C. W. Eriksen \& Schultz, 1979). If CI can generalize to response activation of distractors, then the competing response activated by incompatible distractors should be delayed, leading to a reduction in response competition, and hence a faster target response.

The present study determined whether CI could occur for targets in a selection task and whether CI could generalize to actions associated with distracting stimuli (distractors). Whether or not CI occurs in a selection task and can generalize to actions associated with distractors will indicate whether CI occurs during response selection or at an earlier, response activation stage of processing. The $\mathrm{COH}$ assumes that $\mathrm{CI}$ originates at a level of processing between late perception and early response (Hommel et al., 2001), where memory plays a role in feature-code generation of the action plan (late perception) before the motor programs are selected to execute the action (early response). Thus, the $\mathrm{COH}$ predicts that CI should occur for selected actions, and for actions associated with distractors, because code occupation should delay activation of a response that requires a code that is occupied by a different action plan. If, however, CI occurs only for selected actions, and not for actions associated with distractors, this would suggest that CI originates at a response selection stage.

\section{EXPERIMENTS 1A AND 1B}

Compatibility interference was examined using the Stoet and Hommel (1999) paradigm described earlier, with a 
variant of the Eriksen distractor task (e.g., B. A. Eriksen \& C. W. Eriksen, 1974). Participants were presented an initial stimulus (Stimulus A) that required a sequence of keypress responses to be held in memory. Then an intervening stimulus (Stimulus B) appeared. The target contained in Stimulus B required either the same action hand as Stimulus A or a different action hand. In addition, there were three distractor conditions within Stimulus B. Distractors were either response-compatible with the target or responseincompatible with the target, or no distractors were presented with the target. Participants were instructed to hold the planned response to Stimulus A in memory while they made a speeded response to the target contained in Stimulus B. After responding to Stimulus B, participants were instructed to execute a nonspeeded response to Stimulus A.

If CI occurs in a selection task, the reaction time (RT) to the Stimulus B target should be longer when it requires the same hand as Stimulus A, as opposed to when it requires a different hand. In addition, the typical results found in the Eriksen distractor task suggest that RTs to the intervening target stimulus (Stimulus B) should be longer when it is flanked by incompatible distractors (due to response competition) than when it is flanked by compatible distractors or no distractors. Moreover, if CI can occur for actions associated with distractors, the difference in RTs between action hands (CI effect) should be larger for the incompatible distractor condition than for the no-distractor condition. There should be a greater reduction in Stimulus B RTs in the incompatible distractor condition than in the no-distractor condition when the Stimulus B distractors and Stimulus A use the same action hand (and both the Stimulus B distractors and Stimulus A use a different action hand than does the Stimulus B target). The Stimulus B RTs should be faster in this case because the feature code associated with the target is not occupied and the action feature associated with the incompatible distractors is occupied and is not free to compete with and subsequently delay the target response. The relatively faster target RTs resulting from the occupation of codes needed to activate the distractor response would occur in the incompatible distractor case, but not in the case in which no distractors are presented. If, however, the response activation by the incompatible distractors is not reduced due to code occupation, the reduction in RTs found when the two stimulus events require different action hands should be equivalent to that observed when no distractors (or compatible distractors) are present. That is, there should be no interaction indicating a larger difference between action hand (same or different) when incompatible distractors are present, as opposed to when no distractors are present.

In Experiment 1B, the stimulus onset asynchrony (SOA) for the distractors was changed so that they appeared $100 \mathrm{msec}$ before the onset of the target. This was done to ensure that the response competition evoked by the incompatible distractors was sufficiently large to detect any significant reduction in incompatible distractor activation due to CI. C. W. Eriksen and Schultz (1979) showed that presenting incompatible distractors $100 \mathrm{msec}$ prior to the onset of a target led to increased target RT and error trends relative to when these distractors and the target were presented simultaneously. This finding suggests that presenting the incompatible distractors $100 \mathrm{msec}$ prior to the target allows the distractors to be identified and activate their associated responses earlier, leading to a larger accumulation of incorrect activation, which must be overridden for one to respond correctly to the target. Thus, if response activation by the distractors can be reduced due to code occupation, we should be able to detect this reduction in activation by presenting the distractors prior to target presentation.

\section{Method}

\section{Participants}

A total of 91 undergraduate students from Washington State University participated for optional credit in their psychology courses; 48 participated in Experiment 1A, and 43 participated in Experiment 1B. All participants had at least 20/40 visual acuity, as assessed using a Snellen chart. In Experiment 1A, 17 participants were excluded for not following instructions (moving fingers or tensing muscles during action planning), and 3 participants were excluded for correctly guessing the hypothesis; data are reported for the remaining 28 participants. In Experiment 1B, 14 participants were excluded for not following instructions (moving fingers or tensing muscles during action planning), and 1 participant was excluded for correctly guessing the hypothesis; data are reported for the remaining 28 participants.

\section{Apparatus}

Stimuli were presented on a 17 -in. CRT monitor approximately $41 \mathrm{~cm}$ from the participant in a room painted black. Responses were executed with the left or right index finger by pressing keys on one of two keypads. One keypad was located to the left and the other to the right of the participant's body midline. A distance of $22 \mathrm{~cm}$ separated the keypads, and each keypad was $6 \mathrm{~cm}$ in front of the CRT. Each keypad had three keys $(1 \times 1 \mathrm{~cm}$ in size, separated from the others by $0.2 \mathrm{~cm}$ ) oriented in a vertical array. The key in the middle of each keypad was designated as the home key. A diagram indicating which keypress combinations corresponded with which letters and arrow-asterisk combinations was written on a sheet of paper and placed to the right of the right keypad.

\section{Stimuli and Responses}

All stimuli appeared as white letters and symbols on a black background. A fixation cross (approximately $0.70^{\circ}$ of visual angle) appeared at the center of the CRT screen before and during each trial.

Stimulus A. Stimulus A always appeared above the fixation cross. It consisted of an arrowhead $\left(0.70^{\circ}\right.$ of visual angle $)$ pointing either to the left or to the right (" $<$ " or " $>$ ") and a white asterisk ("* "; $0.70^{\circ}$ of visual angle) that appeared either $0.42^{\circ}$ of visual angle above or $0.28^{\circ}$ of visual angle below the arrowhead. When the asterisk appeared above the arrowhead, the arrowhead was approximately $1.82^{\circ}$ of visual angle above the fixation cross (edge to edge). When the asterisk appeared below the arrowhead, the asterisk was approximately $1.68^{\circ}$ of visual angle above the fixation cross (edge to edge). The arrowhead direction (left or right) indicated the hand with which to respond to the stimulus (left or right). The location of the asterisk, relative to the arrowhead (above/below), indicated the movement direction of the index finger, in relation to the home key. An asterisk positioned above the arrowhead indicated an "above" response (home key, key above the home key, home key), and an asterisk positioned below the arrowhead indicated a "below" response (home key, key below the home key, home key). Note that all responses began and ended by pressing down on the appropriate home key with the index finger. Figure 1 shows the different key- 


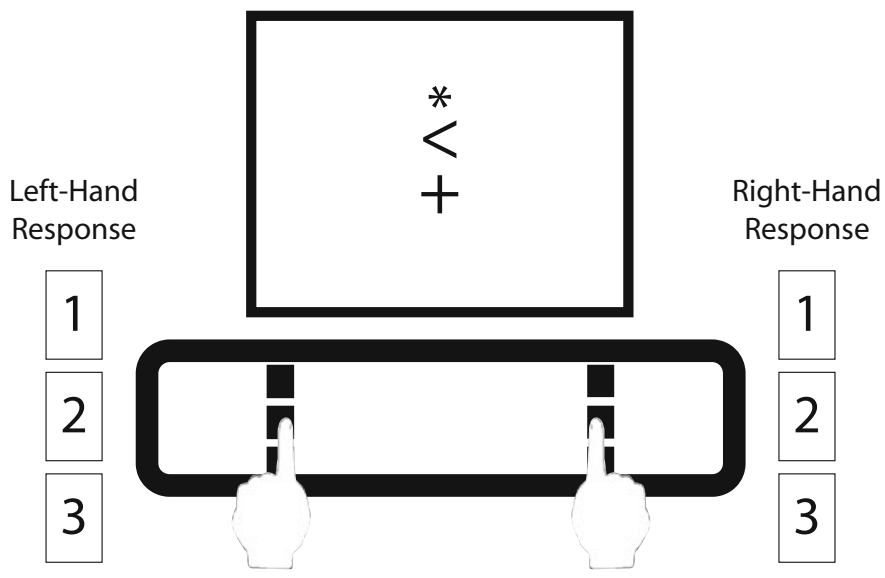

Figure 1. A diagram of the response apparatus used to respond to Stimulus $A$ in Experiments 1 and 2. The left and right response keys are magnified to the right and left, respectively, and they contain numbered keys to aid in describing the different response sequences associated with Stimulus $A$. The arrowhead direction $(<$ or $>$ ) indicated the response hand (left or right) and the asterisk location relative to the arrowhead indicated which keypress sequence to execute. The asterisk above the arrowhead indicated keypresses of 2-1-2, and the asterisk below the arrowhead indicated keypresses of 2-3-2.

press responses corresponding with each of the four stimuli created from the two possible arrowhead directions (right or left) and two possible asterisk locations (above or below the arrowhead).

Stimulus B. Stimulus B consisted of either a single letter or a horizontal array of three letters centered below the fixation cross. Letters consisted of Hs and/or Ss. Each letter subtended approximately $0.84^{\circ}$ of visual angle, with a spatial separation of approximately $0.84^{\circ}$ of visual angle. Participants were instructed to attend and respond to the letter that appeared directly below the fixation cross (target) and to ignore any letters that could occur in adjacent locations to the right and left of the target (distractors). With regard to the different targetdistractor stimulus combinations, a target letter (H or S) could appear alone, without distractors (i.e., $\mathrm{H}$ or $\mathrm{S}$ ); be flanked by two letters identical to the target (i.e., compatible distractors: H H H or S S S); or be flanked by two letters different from the target (i.e., incompatible distractors: S H S or H S H). Participants made a two-choice speeded response on the basis of the identity of the target $(\mathrm{H}$ or $\mathrm{S})$. Half of the participants pressed the home key twice with the left hand if the target letter was an $\mathrm{H}$ and pressed the home key twice with the right hand if the target letter was an S. The other half of the participants had the opposite stimulus-response assignment.

\section{Procedure}

The trial sequence of events for Experiment 1A is shown in Figure 2. At the start of each trial, an initiation screen appeared with a fixation cross and a message that read Press the home keys to continue. When both home keys were pressed simultaneously (with the right and left index fingers), a fixation cross appeared for 1,000 msec. Afterward, Stimulus A appeared above the fixation cross for $2,000 \mathrm{msec}$, followed by a screen that presented the fixation cross alone for 1,250 msec. During this time, participants planned a response to Stimulus A. Stimulus B then appeared below the fixation cross for $250 \mathrm{msec}$ (Experiment 1A), or Stimulus B distractors appeared alone for $100 \mathrm{msec}$ and were followed by the Stimulus B target, for a total target-distractor duration of $250 \mathrm{msec}$ (Experiment 1B). ${ }^{1} \mathrm{~A}$ blank screen appeared after presentation of the Stimulus B target and remained present for $4,750 \mathrm{msec}$, or until a response to Stimulus B was recorded. Participants were instructed to respond as quickly and accurately as possible to the target in Stimulus B as soon as it was presented. RTs to Stimulus B were calculated from the onset of the Stimulus B target to the first keypress detected for Stimulus B. After executing the response to Stimulus B, participants had 5,000 msec to execute the planned response to Stimulus A. Participants were instructed to emphasize accuracy, not speed, when responding to Stimulus A. A single feedback screen indicating the RT and accuracy for Stimulus B and the accuracy for Stimulus A was then presented for $950 \mathrm{msec}$. Afterward, the initiation screen again appeared, indicating the start of a new trial. Participants initiated a new trial by pressing the home keys simultaneously, and the trial sequence was repeated.

Participants were instructed not to execute the planned response (or any part of the planned response) to Stimulus A until after they had perceived and responded to Stimulus B. In addition, they were instructed not to move any fingers, tense any muscles, or use any other external cues to help them remember the planned response sequence to Stimulus A; they were instructed to hold the planned action sequence to Stimulus A in memory only. The experimenter observed each participant during the practice trials, and if a participant had difficulty understanding the order of the two responses, the experimenter would repeat the correct order of the responses and would remain with the participant until the participant's performance indicated that the instructions were understood. If a participant was observed moving fingers or tensing the body on the practice trials during the interval between Stimulus A and Stimulus B presentation, the experimenter reminded the participant not to use external cues to remember the action plan for Stimulus A, but to rely only on a memory representation of the action plan. If the participant continued to use external cues, his or her data were excluded from the analyses.

Two different factors were manipulated. First, the hand required for executing the correct action plan for Stimulus B and Stimulus A was either the same or different. The correct response hand for Stimulus B was determined by the identity of the target letter. The correct response hand required for carrying out an action for Stimulus A was determined by the direction of the arrowhead. Second, Stimulus B contained a target that could be flanked by distractors that were to be ignored. There were three different distractor conditions: no distractors, response-compatible distractors, and response-incompatible distractors. The three different distractor conditions were paired equally across the two possible target letters ( $\mathrm{H}$ or $\mathrm{S})$. 


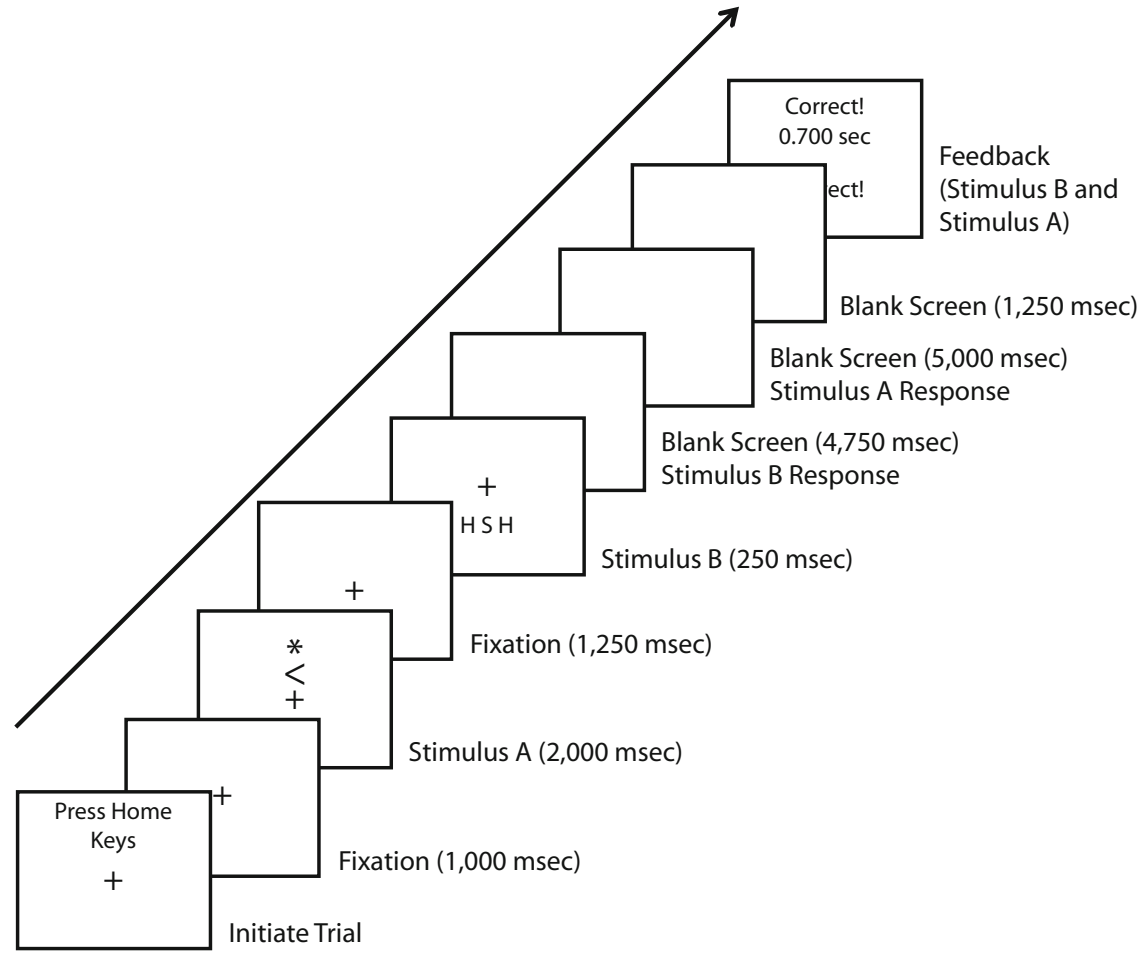

Figure 2. The sequence of trial events in Experiment 1A. The arrow represents the order of events in time.

Participants completed one 90-min session. Each session consisted of 24 practice trials (with an accuracy criterion of $80 \%$ ), with the four possible stimuli for Stimulus A equally paired with the six possible stimuli for Stimulus B (i.e., two possible targets and three distractor conditions), which appeared in a random order, with equal probability of occurrence. Participants then completed eight blocks of 48 experimental trials, with a break at Block 6 . Within each block of 48 trials, 24 trials required the same action hand for Stimuli B and $\mathrm{A}$, and the other 24 trials required different action hands for Stimuli B and A. Action-hand condition (same, different) between Stimuli B and A occurred in a random order, with an equal probability of occurrence, in each block of trials. Also, within each actionhand condition (same, different), one third of the Stimulus B trials contained no distractors (target alone), one third contained compatible distractors, and one third contained incompatible distractors. The different distractor conditions occurred in a random order, with equal probability of occurrence within each action-hand condition within each block.

At the end of the experiment, participants answered three debriefing questions about their task performance during the experimental trials: "What do you think the experiment was about?", "What strategies did you use, if any, to help you respond to the stimuli?", and "Did you move your fingers or tense your muscles when planning your response to the first stimulus (Stimulus A)?" Participants who correctly guessed the hypothesis were excluded from the analyses. Also, participants who reported or were observed moving their fingers over the response keys or tensed muscles on one side of the body during the interval in which a response was to be planned (not executed) and held in memory were excluded from the analyses; those who had engaged in the latter behaviors had violated explicit task instructions.

\section{Results}

For each experiment, the RT analysis for Stimulus $\mathrm{B}$ was restricted to trials in which responses to both
Stimuli B and A were correct. The mean correct RT and percentage of error for Stimulus B for action hand and distractor are shown in the left side of Figure 3 for Experiment $1 \mathrm{~A}$ and in the right side of Figure 3 for Experiment 1B. As is evident in Figure 3, CI due to action-hand similarity was equivalent across the different distractor conditions. The mean percentage of error for Stimulus A for action hand and distractor is presented for each experiment in Table 1. A two-way repeated measures ANOVA with the factors of action hand and distractor was conducted separately on correct RT and percentage of error for Stimulus B and on percentage of error for Stimulus A. All planned comparisons were conducted using Tukey's HSD $(p<.05)$.

\section{Experiment 1A}

Stimulus B. For mean correct RT, there was a significant main effect of action hand $\left[F(1,27)=17.68, M S_{\mathrm{e}}=\right.$ $\left.1,403.33, p<.001, \eta_{\mathrm{p}}^{2}=.396\right]$ and distractor $[F(2,54)=$ $\left.138.65, M S_{\mathrm{e}}=289.70, p<.001, \eta_{\mathrm{p}}^{2}=.837\right]$. The interaction between action hand and distractor was not significant $\left[F(2,54)=2.53, M S_{\mathrm{e}}=174.09, p=.09\right]$, and trends did not suggest a larger difference in RT between action hands (same, different) for the incompatible-distractor condition. These results indicate that responses to Stimulus B were delayed when the Stimulus B target required the same action hand as Stimulus A, as opposed to a different hand (i.e., there was partial feature overlap between the two actions). Thus, our results show that CI, due to action-hand similarity, can be obtained in a selection 

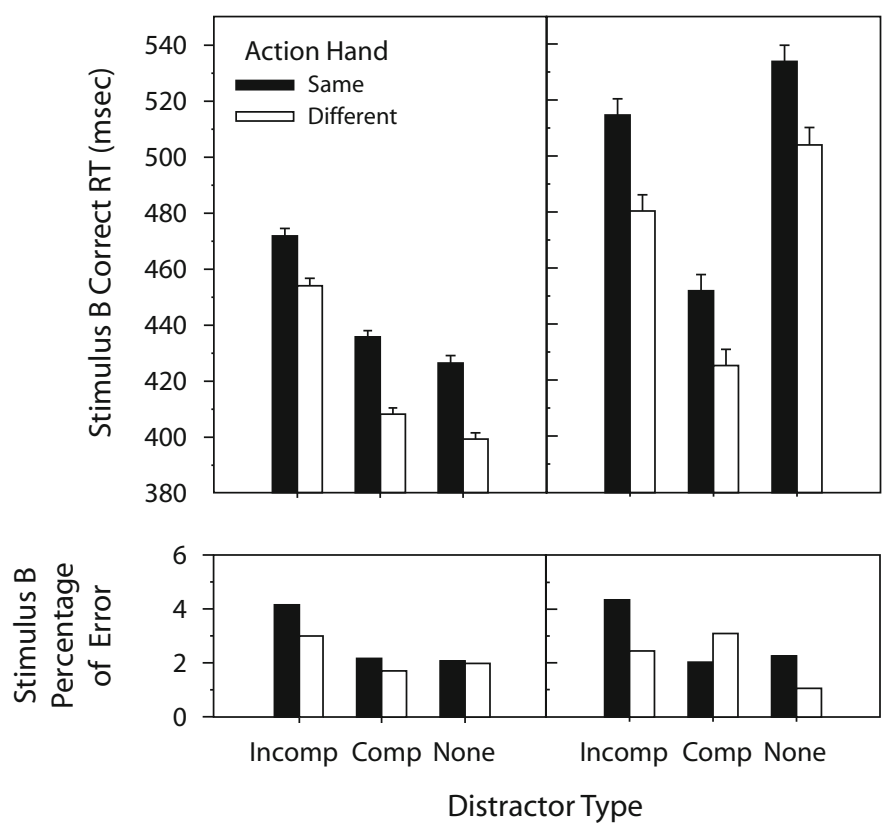

Figure 3. Experiment 1: Correct reaction time (RT) and percentage of error for Stimulus $B$ by action hand (same, different) and distractor type (Incomp $=$ incompatible, Comp $=$ compatible, None $=$ no distractors $)$ in Experiment 1A (left side of chart) and Experiment 1B (right side of chart). Error bars represent one standard error of the mean.

task. Also, planned comparisons showed that responses to Stimulus B were significantly longer when the target was accompanied by incompatible distractors than when the target was accompanied by compatible distractors $(p<.001)$ or no distractors $(p<.001)$. Responses to Stimulus B were also longer when the target was accompanied by compatible distractors than when it was accompanied by no distractors $(p<.05)$. These findings, as well as the size of the distractor effect (incompatible RT of $463 \mathrm{msec}-$ compatible RT of $422 \mathrm{msec}=41 \mathrm{msec}$ ), are consistent with previous research (e.g., B. A. Eriksen \& C. W. Eriksen, 1974; C. W. Eriksen \& Schultz, 1979). Furthermore, the CI effect obtained (i.e., the greater RT for action hand same than for action hand different) was equivalent across the three different distractor conditions. Thus, activation of the response associated with the distractors contained in Stimulus B was not delayed when these distractors shared an action feature (i.e., left or right hand) with the action plan (corresponding to Stimulus A) held in memory. In fact, the trend toward a significant interaction suggests a smaller difference between same and different action hands for the incompatible-distractor than between those for the no-distractor condition. This trend is not consistent with the $\mathrm{COH}$, which would predict a larger difference between the same and different action hands for the incompatible-distractor than between those for the no-distractor condition.

In terms of error rate, there was a significant effect found for distractor $\left[F(2,54)=5.53, M S_{\mathrm{e}}=0.09, p<\right.$ $\left..05, \eta_{\mathrm{p}}^{2}=.170\right]$, indicating that error rates were $2 \%$ higher for targets accompanied by incompatible distractors than for those accompanied by compatible distractors or no distractors $(p \mathrm{~s}<.05)$. There was no significant effect for action hand $\left[F(1,27)=2.89, M S_{\mathrm{e}}=0.05, p>.10\right]$, nor for the interaction between action hand and distractor $\left[F(2,54)=1.22, M S_{\mathrm{e}}<0.04, p=.31\right]$. These results suggest that the RT interpretations above are not due to a speed-accuracy trade-off.

Stimulus A. The average error rate for Stimulus A was $9.3 \%$. There was a significant effect of action hand

Table 1

Mean Percentages of Errors for Stimulus A by Action Hand and Distractor Type for Each Experiment

\begin{tabular}{|c|c|c|c|c|c|c|}
\hline \multirow[b]{3}{*}{ Experiment } & \multicolumn{6}{|c|}{ Action Hand } \\
\hline & \multicolumn{3}{|c|}{ Same } & \multicolumn{3}{|c|}{ Different } \\
\hline & Incompatible & Compatible & None & Incompatible & Compatible & None \\
\hline $1 \mathrm{~A}$ & $12.4^{\mathrm{ad}}$ & $9.2^{\mathrm{a}}$ & $10.3^{\mathrm{e}}$ & $8.4^{\mathrm{bd}}$ & $8.4^{c}$ & $7.0^{\text {bce }}$ \\
\hline 1B & $11.6^{\mathrm{a}}$ & $9.5^{\mathrm{a}}$ & 11.3 & $8.0^{\mathrm{b}}$ & $10.6^{b}$ & 8.4 \\
\hline 2 & 9.8 & 9.3 & 8.6 & 6.9 & 7.3 & 8.6 \\
\hline
\end{tabular}

Note-The within-participants standard error of the mean was 0.01 for all values. Values in the same row that share the same letter superscripts differ at $p<.05$. 
$\left[F(1,27)=14.37, M S_{\mathrm{e}}=0.21, p<.001, \eta_{\mathrm{p}}^{2}=.347\right]$ and distractor $\left[F(2,54)=3.22, M S_{\mathrm{e}}=0.16, p<.05, \eta_{\mathrm{p}}^{2}=\right.$ .107], as well as a significant interaction between action hand and distractor $\left[F(2,54)=4.48, M S_{\mathrm{e}}=0.09, p<.05\right.$, $\left.\eta_{\mathrm{p}}^{2}=.142\right]$ for error rate. Planned comparisons showed greater error rates for the incompatible-distractor than for the no-distractor condition $(p<.05)$, and error rates for these two distractor conditions increased to a similar degree when Stimuli A and B required the same action hand $(p=.55)$. Error rates for the compatible-distractor condition did not vary between action hands $(p=.42)$ (see Table 1). Because these results are not relevant to our hypothesis, they will not be discussed further. It is important, however, that error rates were low for Stimulus A overall, suggesting that the participants had little difficulty holding the response sequence corresponding to Stimulus A in memory.

In summary, both the typical CI effect and the distractor effects found in past research were obtained in this selection task. However, in contrast with the predictions of the $\mathrm{COH}$, we did not find an interaction between action hand and distractor, which would have indicated a larger difference between action hand (same, different) when incompatible distractors were present than when no distractors were present. Thus, response competition by the incompatible distractors was not significantly reduced when these distractors shared an action feature with the planned action held in memory.

\section{Experiment 1B}

Figure 3 (right side) shows that although the distractor effect increased relative to that in Experiment 1A when the distractors appeared $100 \mathrm{msec}$ before the target, the $\mathrm{CI}$ effect remained constant across the different distractor conditions.

Stimulus B. For mean correct RT, there was a significant main effect of action hand $\left[F(1,27)=16.57, M S_{\mathrm{e}}=\right.$ $\left.2,302.56, p<.01, \eta_{\mathrm{p}}^{2}=.380\right]$ and distractor $[F(2,54)=$ $\left.117.67, M S_{\mathrm{e}}=822.26, p<.001, \eta_{\mathrm{p}}^{2}=.813\right]$. The interaction between action hand and distractor was not significant $(F<1)$. In general, these results are comparable to those obtained in Experiment 1A. The responses to Stimulus B were delayed when the Stimulus B target required the same action hand as Stimulus A, as opposed to a different action hand (CI). Also, planned comparisons showed that responses to Stimulus B were significantly longer when the target was accompanied by incompatible, as opposed to compatible, distractors $(p<.001)$. However, in contrast with the findings of Experiment 1A, responses to Stimulus B were significantly faster when distractors were present than when no distractors were present $(p$ s $<$ $.001)$. The faster RTs found when distractors were present can be attributed to a target-alerting effect (see C. W. Eriksen \& Murphy, 1987). Because the distractors always preceded the target SOA by $100 \mathrm{msec}$, the distractors served as a temporal warning signal for the target presentation; this warning signal was absent in the no-distractor condition. Consistent with this interpretation, C. W. Eriksen and Murphy showed that RTs were faster after prestimulus cues that appeared $100 \mathrm{msec}$ prior to a target on a subset of trials than on trials that were not cued, and they attributed the faster RTs to an alerting effect that was triggered by the precue. We also obtained a large distractor effect: RTs were $59 \mathrm{msec}$ longer when the distractors were incompatible than when they were compatible with the target. Moreover, the CI effect obtained (the RT difference between action hand same and action hand different) was equivalent across the three different distractor conditions. Thus, similar to the findings in Experiment 1A, activation of the distractors was not delayed when the distractors shared an action feature (i.e., response hand) with the action plan held in memory.

The error results for Stimulus B confirmed this conclusion. There was a significant main effect of distractor $\left[F(2,54)=7.45, M S_{\mathrm{e}}<0.06, p<.01, \eta_{\mathrm{p}}^{2}=.216\right]$ and a significant interaction between action hand and distractor $\left[F(2,54)=5.82, M S_{\mathrm{e}}<0.06, p<.01, \eta_{\mathrm{p}}^{2}=.177\right]$. The main effect of action hand was not significant $[F(1,27)=$ $\left.2.05, M S_{\mathrm{e}}<0.09, p=.16\right]$. Planned comparisons showed that error rates were greater for the incompatible-distractor than for the no-distractor condition $(p<.05)$, and error rates for these two distractor conditions increased to a similar degree when Stimuli A and B required the same action hand, as opposed to different action hands ( $p=$ .20). Also, error rates for the compatible-distractor condition did not vary between action hands $(p=.17)$. These results indicate that the RT interpretations above are not due to a speed-accuracy trade-off.

Stimulus A. The average error rate for Stimulus A was $10.0 \%$. There was a significant main effect of action hand $\left[F(1,27)=4.40, M S_{\mathrm{e}}<0.32, p<.05, \eta_{\mathrm{p}}^{2}=.140\right]$ and a significant interaction between action hand and distractor $\left[F(2,54)=6.02, M S_{\mathrm{e}}<0.15, p<.01, \eta_{\mathrm{p}}^{2}=.182\right]$ for error rate. The main effect of distractor was not significant $(F<1)$ for error rate (see Table 1$)$. Planned comparisons showed that error rates increased to a similar degree in the incompatible- and no-distractor conditions when Stimuli A and B required the same action hand as opposed to different action hands $(p<.01)$; however, Stimulus A error rates did not vary by action hand in the compatible-distractor condition $(p=.40)$. These results are similar to those found in Experiment 1A. Also, the low error rate found for Stimulus A indicates that participants had little difficulty recalling the planned action held in memory.

\section{Comparison Between Experiments $1 \mathrm{~A}$ and 1B}

Data between Experiments 1A and 1B were compared to determine whether early presentation of the distractors in Experiment 1B increased the response strength of the distractors relative to Experiment $1 \mathrm{~A}$ and to determine whether the CI effects found across distractor conditions were the same in both experiments. A mixed-design ANOVA, with the between-participants factor of experiment and within-participants factors of action hand and distractor, was conducted separately on the mean correct RT and percentage of errors for Stimulus B.

The only significant effects involving the factor of experiment included a main effect of experiment $[F(1,54)=$ $\left.5.2, p<.05, M S_{\mathrm{e}}=45,463.0, \eta_{\mathrm{p}}^{2}=.088\right]$ and an interac- 
tion between experiment and distractor type $[F(2,108)=$ $\left.112.89, M S_{\mathrm{e}}=555.98, p<.001, \eta_{\mathrm{p}}^{2}=.676\right]$ for RT. Planned comparisons revealed that the interaction between experiment and distractor type was partly due to differences between the incompatible- and the compatibledistractor conditions across experiments $(p<.01)$. Thus, as predicted, having the distractors precede the target by $100 \mathrm{msec}$ in Experiment $1 \mathrm{~B}$ increased the response strength of the distractors (i.e., the distractor effect). The distractor effect (incompatible RT - compatible RT) significantly increased by $18 \mathrm{msec}$ in Experiment 1B (see Figure 3). This interaction was also due to the larger RT found for the single-letter (no-distractor) condition relative to those found for the compatible- $(p<.001)$ and incompatible- $(p<.001)$ distractor conditions in Experiment 1B (i.e., due to the alerting effect present in Experiment 1B). Moreover, increasing the distractor effect in Experiment 1B relative to that in Experiment 1A did not result in a larger CI effect for the incompatible- relative to the no-distractor condition. The three-way interaction among the factors of experiment, action hand, and distractor was not significant for RT $[F(2,108)=1.27$, $\left.M S_{\mathrm{e}}=478.98, p=.28\right]$, and the significant trend found for accuracy $\left[F(2,108)=3.0, M S_{\mathrm{e}}=0.05, p=.054\right]$ was not due to accuracy differences involving incompatible distractors. The trend toward a significant three-way interaction for accuracy was due to a $1.5 \%$ decrease in accuracy found for Experiment 1B relative to that found for Experiment 1A for the action hand different condition when compatible distractors were present. These results confirm that the CI effect and the distractor effect independently influenced target response performance in both experiments.

\section{Discussion}

The typical CI effect and distractor effects found in past research were obtained in both experiments. The CI effect, which was based on action-hand similarity, was consistent across distractors (incompatible, compatible, or none) flanking the target. Also, the degree of the CI effect remained unchanged when the response activation of the distractors was increased by presenting the distractors $100 \mathrm{msec}$ before the target (yielding a distractor effect of $59 \mathrm{msec}$ ), as opposed to simultaneously with the target (yielding a distractor effect of $41 \mathrm{msec}$ ). The 59-msec distractor effect obtained when the distractors preceded the target is comparable to the 40 - to 50 -msec distractor effects reported in previous research, in which the distractors preceded or were made larger than the target (e.g., C. W. Eriksen \& Schultz, 1979). This result suggests that the response activation by the incompatible distractors should have been large enough to detect whether CI occurred for distractor-response activation - at least in terms of the typical distractor-response activation found in visual selection tasks.

Thus, in contrast with the $\mathrm{COH}$, Experiment 1 showed that CI did not generalize to distractor-response activation. The $\mathrm{COH}$ predicts that when a distractor has a response associated with the same hand as the action plan held in memory, the activation associated with the distractor should be delayed. This is because the code required to activate this distractor's response should be occupied by the action plan held in memory and, hence, should be less available. A delay in distractor-response activation should reduce the impact an incompatible distractor has on the target response. If this had occurred, Experiment 1 should have revealed an interaction between action hand and distractor, indicating a larger difference between action hand (same, different) when incompatible distractors were present, as opposed to when no distractors were present. The failure to find such evidence, particularly in the case in which the distractor effect was large (Experiment 1B), indicates that the response activation by the incompatible distractors was not delayed when they shared an action feature (i.e., left or right hand) with the action plan occupied in memory. Instead, it appears that the feature codes needed to activate the distractor response were readily available. Because CI only occurred for selected actions, and not for actions associated with distractors, this suggests that CI occurs during response selection.

\section{EXPERIMENT 2}

In the selection task used in Experiment 1, there were only two possible targets $(\mathrm{H}, \mathrm{S})$ and two possible distractors $(\mathrm{H}, \mathrm{S})$. It could be argued that with such a simple design (only one target mapped to each response) and many trials, response selection of the target could occur almost immediately, which may require very little response activation by the target. As a consequence, this may result in little response activation by the distractors prior to target response selection. This, however, is unlikely for two reasons. First, the distractor effects in Experiment 1, particularly when the distractors preceded the target, were large. Second, psychophysiological research has shown that incompatible distractors flanking the target can activate their associated response (i.e., with the right or left hand) when using the same target (and distractor) sets or set sizes as those used in Experiment 1. For example, Coles et al. (1985) showed early, incorrect activity over motor cortex (which was based on the lateralized readiness potential, LRP) when the letter distractors (e.g., Hs) flanking the target were incompatible with the letter target (e.g., S). Also, Wild-Wall, Falkenstein, and Hohnsbein (2008) showed similar early, incorrect activity over motor cortex (which was based on the LRP) for incompatible distractors flanking the target when the target and distractors consisted of right- and left-pointing arrowheads. Taken together, these findings suggest that response activation by the incompatible distractors in Experiment 1 should have been sufficient for detecting whether CI occurred for these distractors.

Although it is unlikely that the results obtained in Experiment 1 were due to insufficient response activation by the distractors (due to quick selection of the target response), it is important to ensure that the results obtained in Experiment 1 can generalize to a more complex selection task, in which response selection requires more time. To test this, we increased the target (and distractor) 
set size in Experiment 2. Increasing the target set should create a larger demand on memory and lengthen response selection times (for a review, see Kornblum, Hasbroucq, \& Osman, 1990). However, increasing the target set will decrease stimulus certainty, which, in turn, may decrease the distractor effect. This is because a decrease in stimulus certainty should require longer perceptual evaluation in order to resolve critical stimulus features, to begin identifying (or categorizing) the stimulus. This should also delay response activation associated with the stimulus and, hence, response selection time. Because attention may speed perceptual evaluation of the target, the added delay in perceptual evaluation for the distractors should delay their response activation and the amount of response activation accumulated before target response selection.

\section{Method}

\section{Participants}

Thirty-six undergraduate students from Washington State University participated for optional credit in their psychology courses. Seven participants were excluded for not following instructions (moving fingers or tensing muscles during action planning), and 1 participant was excluded for correctly guessing the hypothesis. Data are reported for the remaining 28 participants.

\section{Apparatus, Stimuli, and Procedure}

The apparatus, stimuli, and procedures were identical to those in Experiment 1A, except as follows. For Stimulus B, the number of stimuli and the stimulus-to-response mapping ratio were increased. Stimulus B targets and distractors consisted of the letters $\mathrm{H}, \mathrm{K}, \mathrm{S}$, and $\mathrm{C}$. Two of the letters $(\mathrm{H}, \mathrm{K})$ were mapped to one response hand (e.g., left), and the other two letters (S, C) were mapped to the other response hand (e.g., right). There were three main types of targetdistractor conditions that were formed on the basis of these stimuli, so that a target letter $(\mathrm{H}, \mathrm{K}, \mathrm{S}, \mathrm{C})$ could either appear alone, without distractors (i.e., H, K, S, or C); be flanked by two letters with the same response mapping as the target (i.e., compatible distractors: H H H, K H K, K K K, H K H, S S S, C S C, C C C, or S C S); or be flanked by two letters with a different response mapping than the target (i.e., incompatible distractors: S H S, C H C, S K S, C K C, $\mathrm{H} \mathrm{S} \mathrm{H,} \mathrm{K} \mathrm{S} \mathrm{K,} \mathrm{H} \mathrm{C} \mathrm{H,} \mathrm{or} \mathrm{K} \mathrm{C} \mathrm{K).} \mathrm{Half} \mathrm{of} \mathrm{the} \mathrm{participants} \mathrm{pressed}$ the home key twice with the left hand if the central letter (target) was an $\mathrm{H}$ or a $\mathrm{K}$ and pressed the home key twice with the right hand if the target was an S or a C. The other half of the participants had the opposite stimulus-response assignment.

There were four blocks of 96 experimental trials (with a break between Blocks 2 and 3). Similar to Experiment 1, action-hand condition (same, different) between Stimuli B and A occurred in a random order, with an equal probability of occurrence in each block of trials. Also, within each action-hand condition (same, different), one third of the Stimulus B trials contained no distractors (target alone), one third contained compatible distractors, and one third contained incompatible distractors. Finally, the different distractor conditions occurred in a random order, with equal probability of occurrence within each action-hand condition within each block.

\section{Results and Discussion}

The RT analysis for Stimulus B was restricted to trials on which responses to both Stimuli B and A were correct. The mean correct RT and percentage of error for Stimulus B by action hand and distractor are shown in Figure 4. Consistent with the findings of Experiment 1,
Figure 4 shows that $\mathrm{CI}$ due to action-hand similarity was equivalent across the different distractor conditions. The mean percentage of error for Stimulus A by action hand and distractor is presented in Table 1. A two-way repeated measures ANOVA with the factors of action hand and distractor was conducted separately on correct RT and percentage of error for Stimulus B and on percentage of error for Stimulus A. All planned comparisons were conducted using Tukey's HSD $(p<.05)$.

Stimulus B. For mean correct RT, there was a significant main effect of action hand $\left[F(1,27)=35.31, M S_{\mathrm{e}}=\right.$ $\left.871.28, p<.001, \eta_{\mathrm{p}}^{2}=.567\right]$ and distractor $[F(2,54)=$ $\left.48.73, M S_{\mathrm{e}}=593.40, p<.001, \eta_{\mathrm{p}}^{2}=.643\right]$. Moreover, the interaction between action hand and distractor was not significant $\left[F(2,54)=1.09, M S_{\mathrm{e}}=352.17, p=.34\right]$. These results are comparable to those obtained in Experiment 1. Stimulus B responses were delayed when the Stimulus B target required the same hand as Stimulus A. Also, Stimulus $\mathrm{B}$ responses were longest when the distractors were incompatible, intermediate when the distractors were compatible, and fastest when no distractors were present $(p \mathrm{~s}<.001)$. Moreover, the CI effect was equivalent across the three different distractor conditions. Thus, activation of the distractors was not delayed when the distractors shared an action feature (i.e., response hand) with the action plan held in memory.

The error results for Stimulus B confirmed this conclusion. There was a significant main effect of distractor $\left[F(2,54)=11.72, M S_{\mathrm{e}}=0.07, p<.001, \eta_{\mathrm{p}}^{2}=.303\right]$, indicating that error rate was greater in the incompatible- than in the compatible- and no-distractor conditions $(p \mathrm{~s}<$
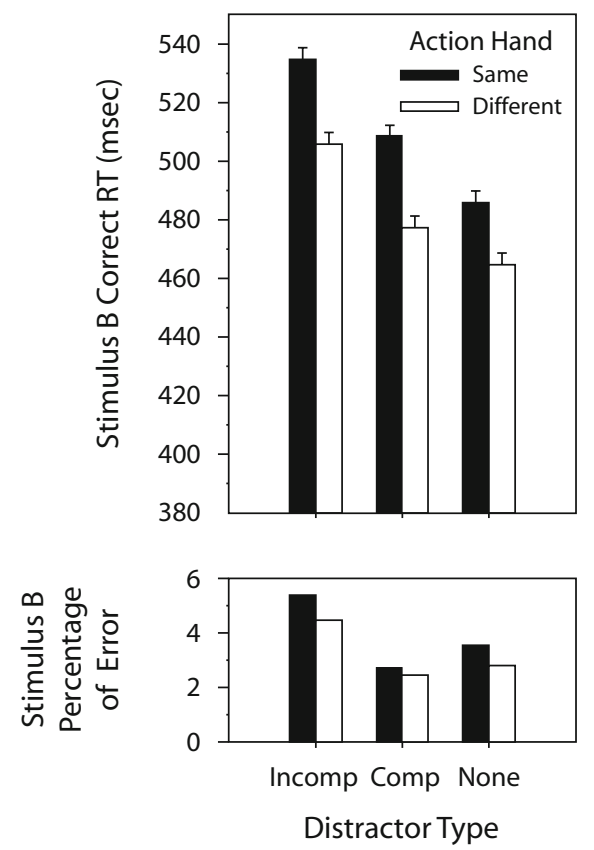

Figure 4. Experiment 2: Correct RT and percentage of error for Stimulus $B$ by action hand (same, different) and distractor type (Incomp $=$ incompatible, Comp $=$ compatible, None $=$ no distractors). Error bars represent one standard error of the mean. 
$.01)$, which did not differ in error rate $(p=.13)$. The main effect of action hand $\left[F(1,27)=1.27, M S_{\mathrm{e}}=0.14, p=\right.$ .27] and the interaction between action hand and distractor $(F<1)$ were not significant.

Stimulus A. The average error rate for Stimulus A was 8.4\%, which is comparable to that in Experiment 1. There was no significant effect found for action hand $[F(1,27)=$ $\left.2.35, M S_{\mathrm{e}}=0.55, p=.14\right]$, distractor $(F<1)$, or the interaction between these factors $\left[F(1,54)=2.29, M S_{\mathrm{e}}=\right.$ $0.10, p=.11]$ on error rate.

\section{Comparison Between Experiments 2 and $1 \mathrm{~A}$}

Data from Experiments 2 and 1A were compared in order to determine whether the CI effects found across distractor conditions were the same in both experiments, even though the target set for Stimulus B was larger and the target-distractor conditions were more variable in Experiment 2. A mixed-design ANOVA with the betweenparticipants factor of experiment and within-participants factors of action hand and distractor was conducted separately on the mean correct RT and percentage of errors for Stimulus B.

The only significant effects involving the factor of experiment included a main effect of experiment $[F(1,54)=$ $\left.4.85, M S_{\mathrm{e}}=70,808.90, p<.05, \eta_{\mathrm{p}}^{2}=.082\right]$ and an interaction between experiment and distractor type $[F(2,108)=$ 3.08, $\left.M S_{\mathrm{e}}=441.55, p<.05, \eta_{\mathrm{p}}^{2}=.054\right]$ for RT. These findings indicate that Stimulus B RTs were slower overall in Experiment 2 than in Experiment 1A. Also, the distractor effect was significantly smaller in Experiment $2(M=$ $27 \mathrm{msec}$ ). These findings confirm that increasing the number of stimuli mapped to a response leads to longer RTs (e.g., Kornblum et al., 1990) and can reduce the distractor effect. Also, the lack of a significant two-way interaction between the factors of action hand and distractor suggests that the CI effect and the distractor effect independently influenced target response performance. Moreover, the lack of a three-way interaction between experiment, action hand, and distractor indicates that the size of the CI effect was the same as that obtained in Experiment 1A, even though the larger target set size in Experiment 2 delayed target selection time and reduced the distractor effect. This latter finding confirms that the CI effect and distractor effect independently influenced target response selection.

\section{GENERAL DISCUSSION}

This study showed that CI can occur in a selection task. That is, if a target item that is selected among other distracting items requires the same hand as an action plan held in memory (both actions share a common feature code), a response delay is observed for that selected target. Also, this study showed that in a selection task, CI does not generalize to distracting stimuli that activate the same hand (a common feature code) with the planned action held in memory. There was no evidence that response activation associated with the distractors was delayed when they shared an action feature with the action plan in memory. Presenting the distractors either simultaneously
(Experiments 1A and 2) or prior to the target stimulus (Experiment 1B) and increasing the stimulus-response mapping ratio (Experiment 2) yielded the same conclusion. CI occurred only for the selected action, and not for an action that was activated but not selected; this suggests that $\mathrm{CI}$ is due to a delay in response selection, as opposed to response activation.

These findings pose a problem for the $\mathrm{COH}$ proposed to account for CI. According to the $\mathrm{COH}, \mathrm{CI}$ is due to a delay in accessing, and hence activating, a feature code that is bound and occupied by the action plan held in memory until this action plan is executed or abandoned (e.g., Hommel et al., 2001; Stoet \& Hommel, 1999). The feature codes occupied by the action plan in memory are assumed to be somewhat encapsulated in this state (Tipper, 2001). Thus, if an intervening event requires the use of one of the feature codes bound and occupied by the action plan held in memory, the feature codes that compose this action plan must be briefly disassembled in order to access the needed code for the new event. This process will take time and should result in response activation delays for the intervening event. However, because we found that CI does not delay response activation associated with distractor stimuli contained in the intervening event, this suggests that the feature codes associated with the distractor response were readily available for response activation. This finding indicates that code occupation delays neither access to a feature code nor the activation of a feature code (i.e., at a processing stage prior to "early response"; Hommel et al., 2001). It also suggests that $\mathrm{CI}$ is not due to feature code inhibition (Caessens \& Vandierendonck, 2002), because the inhibition account would predict activation delays for codes that are inhibited, including those response codes associated with distractor stimuli.

CI was found only for actions corresponding to the selected response. Thus, if CI is due to code occupation or inhibition, code occupation or inhibition must somehow delay response selection without delaying response activation. One way in which response selection can be delayed without delaying response activation is by elevating the response selection threshold for the action plan that requires an occupied (or inhibited) code relative to an action plan that does not require an occupied code. Such an assumption could easily be incorporated into the $\mathrm{COH}$. A second possibility is that the occupied code(s) may be available for stimulus-triggered activation (and, hence, these occupied codes are not completely encapsulated), but selection of the intervening response is delayed due to the added time needed to disassemble occupied codes for constructing the new selected action plan. A third possibility is that the response selection threshold is elevated under conditions in which two or more action plans share similar feature codes (Grice, Bouroughs, \& Canham, 1984); action code similarity between two different action plans may elevate the response selection threshold required for selecting the correct action for the current event.

This latter possibility does not attribute CI to code occupation or inhibition. It assumes that CI results from 
confusability between two or more action plans that share similar feature codes (cognitive codes), which, in turn, can delay selection of the action plan relevant to the current event. That is, if two action plans contain similar feature codes, selecting the correct action plan should be more difficult and hence take longer than when the two action plans do not share feature codes (similar to two-choice discrimination tasks that show delayed responding when the stimuli that are mapped to different responses contain similar perceptual features, relative to when they do not; B. A. Eriksen \& C. W. Eriksen, 1974). This selection account is amenable with the $\mathrm{CCH}$, from which the $\mathrm{COH}$ is derived, but assumes that $\mathrm{CI}$ is due to selection delays resulting from confusability between actions or events that share similar feature codes.

This selection explanation for CI can account for our findings and for other CI effects reported in the literature. For example, it can account for the finding that CI is greater when feature code overlap between two action plans increases (Stoet \& Hommel, 1999), since confusability between the action plans would also increase and hence delay selection of the correct action. It also predicts facilitative priming for an action plan that has complete feature code overlap with an action plan currently held in memory (see Hommel et al., 2001; Stoet \& Hommel, 1999), because only one action plan would be represented (available for selection), and this plan would be available earlier for activating a response than would a more recently constructed action plan. Finally, this selection account predicts that recognition of a briefly presented perceptual stimulus that shares a feature code (cognitive code) with a planned action currently held in memory would be delayed, leading to more recognition errors, relative to when these two events do not share codes (e.g., see results by Müsseler \& Hommel, 1997a, 1997b; Müsseler \& Wühr, 2002; Wühr \& Müsseler, 2001; for a review, see Hommel et al., 2001). This recognition delay would be attributed to a delay in selecting the correct representation corresponding to the relevant stimulus event when the two events share similar codes and are therefore highly confusable. Unlike the $\mathrm{COH}$ account, this selection account is similar to that proposed to account for delays in stimulus selection (see, e.g., Duncan \& Humphreys, 1989), as well as in response selection (see, e.g., B. A. Eriksen \& C. W. Eriksen, 1974), when stimuli share similar perceptual features, as opposed to when they do not. However, because this account makes similar predictions to a code occupation account that attributes $\mathrm{CI}$ to a response selection delay, it will be difficult to test these two alternatives.

In summary, we showed that executing a response based on a task-relevant stimulus that must be selected among other stimuli is delayed when this task-relevant stimulus shares an action feature with an action plan currently held in memory (e.g., both actions require the same hand). Our findings suggest that if we plan a sequence of actions required, for example, to turn on a car air conditioner with our right hand - but before executing this action, we retrieve our sunglasses, which are sliding among other items across the dashboard toward us - it may take us longer to retrieve the sunglasses with the right hand than with the left hand. This delay, the CI, is attributed to a response selection delay, as opposed to a response activation delay, in which the intervening action and the action held in memory share similar feature codes. Thus, if code occupation is the mechanism responsible for these selection delays, our results show that the occupied codes are not encapsulated in a way that prevents stimulustriggered response activation.

\section{AUTHOR NOTE}

Correspondence concerning this article should be addressed to L. R. Fournier, Department of Psychology, Washington State University, Pullman, WA 99164-4820 (e-mail: 1fournier@wsu.edu).

\section{REFERENCES}

Caessens, B., \& VAndierendonck, A. (2002). Inhibition and blindness to response-compatible stimuli: A reappraisal. Acta Psychologica, 111, 45-57.

Coles, M. G. H., Gratton, G., Bashore, T. R., Eriksen, C. W., \& Donchin, E. (1985). A psychophysiological investigation of the continuous flow model of human information processing. Journal of Experimental Psychology: Human Perception \& Performance, 11, 529-553.

Duncan, J., \& Humphreys, G. W. (1989). Visual search and stimulus similarity. Psychological Review, 96, 433-458.

Eriksen, B. A., \& ERIKSEN, C. W. (1974). Effects of noise letters upon the identification of a target letter in a nonsearch task. Perception \& Psychophysics, 16, 143-149.

Eriksen, C. W., \& Murphy, T. D. (1987). Movement of attentional focus across the visual field: A critical look at the evidence. Perception \& Psychophysics, 42, 299-305.

Eriksen, C. W., \& Schultz, D. W. (1979). Information processing in visual search: A continuous flow conception and experimental results. Perception \& Psychophysics, 25, 249-263.

Gallese, V., Fadiga, L., Fogassi, L., \& Rizzolatti, G. (1996). Action recognition in the premotor cortex. Brain, 119, 593-609.

Grice, G. R., Bouroughs, J. M., \& CANhaM, L. (1984). Temporal dynamics of associative interference and facilitation produced by visual context. Perception \& Psychophysics, 36, 499-507.

Hommel, B., Müsseler, J., Aschersleben, G., \& Prinz, W. (2001). The theory of event coding (TEC): A framework for perception and action planning. Behavioral \& Brain Sciences, 24, 849-878.

JeAnnerod, M. (1997). The cognitive neuroscience of action. Cambridge, MA: Blackwell.

Keele, S. W., Cohen, A., \& Ivry, R. (1990). Motor programs: Concepts and issues. In M. Jeannerod (Ed.), Attention and performance XIII: Motor representation and control (pp. 77-110). Hillsdale, NJ: Erlbaum.

Kornblum, S., Hasbroucq, T., \& Osman, A. (1990). Dimensional overlap: Cognitive basis for stimulus-response compatibility-A model and taxonomy. Psychological Review, 97, 253-270.

Müsseler, J., \& Hommel, B. (1997a). Blindness to response-compatible stimuli. Journal of Experimental Psychology: Human Perception \& Performance, 23, 861-872.

Müsseler, J., \& Hommel, B. (1997b). Detecting and identifying response-compatible stimuli. Psychonomic Bulletin \& Review, 4, 125-129.

MüSSElER, J., \& WüHR, P. (2002). Response-evoked interference in visual encoding. In W. Prinz \& B. Hommel (Eds.), Attention and performance XIX: Common mechanisms in perception and action (pp. 520537). New York: Oxford University Press.

Prinz, W. (1990). A common coding approach to perception and action. In O. Neumann \& W. Prinz (Eds.), Relationships between perception and action (pp. 167-201). Berlin: Springer.

Prinz, W. (1997). Perception and action planning. European Journal of Cognitive Psychology, 9, 129-154.

Sakata, H., Taira, M., Murata, A., \& Mine, S. (1995). Neural mecha- 
nisms of visual guidance of hand actions in the parietal cortex of the monkey. Cerebral Cortex, 5, 429-438.

Stoet, G., \& Hommel, B. (1999). Action planning and the temporal binding of response codes. Journal of Experimental Psychology: Human Perception \& Performance, 25, 1625-1640.

Stoet, G., \& Hommel, B. (2002). Interaction between feature binding in perception and action. In W. Prinz \& B. Hommel (Eds.), Attention and performance XIX: Common mechanisms in perception and action (pp. 538-552). New York: Oxford University Press.

Taira, M., Mine, S., Georgopoulos, A. P., Murata, A., \& SaKata, H. (1990). Parietal cortex neurons of the monkey related to the visual guidance of hand movements. Experimental Brain Research, 83, 29-36.

TIPPER, S. P. (2001). Does negative priming reflect inhibitory mechanisms? A review and integration of conflicting views. Quarterly Journal of Experimental Psychology, 54A, 321-343.

Wiediger, M., \& Fournier, L. R. (in press). An action sequence withheld in memory can delay execution of visually guided actions: The generalization of response compatibility interference. Journal of Experimental Psychology: Human Perception \& Performance.
Wild-Wall, N., Falkenstein, M., \& Hohnsbein, J. (2008). Flanker interference in young and older participants as reflected in eventrelated potentials. Brain Research, 1211, 72-84.

Wühr, P., \& Müsseler, J. (2001). Time course of the blindness to response-compatible stimuli. Journal of Experimental Psychology: Human Perception \& Performance, 27, 1260-1270.

\section{NOTE}

1. The interstimulus interval (ISI) between the onset of Stimulus A and the onset of Stimulus B in Experiment 1B was the same as that in Experiment $1 \mathrm{~A}(1,250 \mathrm{msec})$, but the interval between the onset of Stimulus A and onset of the target corresponding to Stimulus B was increased by $100 \mathrm{msec}$, creating a target ISI of $1,350 \mathrm{msec}$.

(Manuscript received September 19, 2007; revision accepted for publication June 26, 2008.) 